Bull. Austral. Math. Soc.

$11 \mathrm{G} 05,11 \mathrm{R} 58$

VoL. 58 (1998) [353-357]

\title{
INTEGRAL POINTS ON ELLIPTIC CURVES OVER FUNCTION FIELDS OF POSITIVE CHARACTERISTIC
}

\author{
Amílcar Pacheco
}

Let $K$ be a one variable function field of genus $g$ defined over an algebraically closed field $k$ of characteristic $p>0$. Let $E / K$ be a non-constant elliptic curve. Denote by $M_{K}$ the set of places of $K$ and let $S \subset M_{K}$ be a non-empty finite subset .

Mason in his paper "Diophantine equations over function fields" Chapter VI, Theorem 14 and Voloch in "Explicit p-descent for elliptic curves in characteristic $p$ " Theorem 5.3 proved that the number of $S$-integral points of a Weiertrass equation of $E / K$ defined over $R_{S}$ is finite. However, no explicit upper bound for this number was given. In this note, under the extra hypotheses that $E / K$ is semi-stable and $p>3$, we obtain an explicit upper bound for this number for a certain class of Weierstrass equations called $S$-minimal.

\section{INTRODUCTION}

The paper is organised as follows. In Section 2 we introduce some preliminaries on the canonical height and torsion points of $E$. In Section 3 we show our main result on $S$-integral points.

\section{Preliminaries}

Let $\widehat{h}: E(\bar{K}) \rightarrow \mathbb{R}$ be the canonical height of $E$. Given a place $\mathfrak{p}$ of $K$, let $v_{\mathfrak{p}}$ be the normalised valuation of $K$ corresponding to $\mathfrak{p}, K_{\mathfrak{p}}$ the completion of $K$ with respect to $v_{\mathfrak{p}}$ and $\lambda_{\mathfrak{p}}: E\left(K_{\mathfrak{p}}\right) \rightarrow \mathbb{R}$ the Néron function associated to $\mathfrak{p}$ (see [8, Chapter VI]).

Suppose that $E / K$ is semi-stable. Let $X$ be a smooth irreducible projective curve defined over $k$ with function field $K$. Denote by $\varphi_{\mathcal{E}}: \mathcal{E} \rightarrow X$ the semi-stable minimal model of $E / K$. Let $j_{\mathcal{E}}: X \rightarrow \mathbb{P}_{k}^{1}$ be the $j$-map induced by $\varphi_{\mathcal{E}}$ and $p^{e}$ its inseparable degree. In the sequel we regard $j_{\mathcal{E}}$ as an element of $K$.

Goldfeld and Szpiro in [2, Proposition 11] gave an explicit version of a Theorem of Manin in which $\widehat{h}$ is computed in terms of an intersection number in $\mathcal{E}$. This allows the

Received 27th January, 1998

This work was partially supported by CNPq research grant number 300896/91-3 and Pronex, Brazil.

Copyright Clearance Centre, Inc. Serial-fee code: 0004-9729/98 \$A2.00+0.00. 
decomposition $\widehat{h}(P)=\sum_{\mathfrak{p} \in M_{K}} \lambda_{\mathfrak{p}}(P)$ and reduces the problem of finding a lower bound for the canonical height of points $P$ of infinite order of $E$ to bounding Néron's functions at $P$. The main ingredient to obtain a global result is the following lemma due to Hindry and Silverman.

LEMma 1. [4, Proposition 1.2] Let $\mathfrak{p} \in M_{K}$ be such that $v_{\mathfrak{p}}\left(j_{\mathcal{E}}\right)<0$. For any distinct points $P_{0}, \cdots, P_{N} \in E\left(K_{\mathfrak{p}}\right)$ we have $\sum_{i \neq l} \lambda_{\mathfrak{p}}\left(P_{i}-P_{l}\right) \geqslant\left((N+1)^{2} / 12 v_{\mathfrak{p}}\left(j_{\mathcal{E}}^{-1}\right)\right)-$ $\left((N+1) v_{\mathfrak{p}}\left(j_{\mathcal{E}}^{-1}\right) / 12\right)$.

DEFINITION 2: Let $\mathfrak{D}_{E / K}$ be the minimal discrimimant of $E / K$ and $\mathfrak{F}_{E / K}$ its conductor. Denote $d_{E / K}=\operatorname{deg}\left(\mathfrak{D}_{E / K}\right)$ and $f_{E / K}=\operatorname{deg}\left(\mathfrak{F}_{E / K}\right)$. Let $\sigma_{E / K}=d_{E / K} / f_{E / K}$ be the Szpiro's ratio of $E / K$. Since $E / K$ is semi-stable, $d_{E / K}=\operatorname{deg}\left(j_{\mathcal{E}}\right)=\left[K: k\left(j_{\mathcal{E}}\right)\right]$.

Convention. Given a finite set $T$ we denote by $|T|$ its cardinal.

Proposition 3. The set $\mathcal{S}_{\sigma}=\left\{P \in E(K) ; \widehat{h}(P) \leqslant d_{E / K} \sigma_{E / K}^{-2} / 96\right\}$ has at most $2 \sigma_{E / K}^{2}$ elements.

Proof: Suppose that $\left|\mathcal{S}_{\sigma}\right|>2 \sigma_{E / K}^{2}$. Let $N \geqslant 1$ be any integer such that $2 \sigma_{E / K}^{2}<$ $N+1 \leqslant\left|\mathcal{S}_{\sigma}\right|$. Let $\mathfrak{p} \in M_{K}$ be such that $v_{\mathfrak{p}}\left(j_{\mathcal{E}}\right) \geqslant 0$. It follows from [5, Chapter XI, Theorem 5.1] that for any $P \in E(K), \lambda_{\mathfrak{p}}(P) \geqslant 0$. Given $P_{0}, \cdots, P_{N} \in E(\bar{K})$, let $H=$ $\max _{0 \leqslant i \leqslant N} \widehat{h}\left(P_{i}\right)$. It follows from the triangle inequality that $H \geqslant(1 /(4 N(N+1))) \sum_{i \neq l} \widehat{h}\left(P_{i}-\right.$ $\left.P_{l}\right)$. Hence, Proposition 1 implies $H \geqslant(1 /(48 N)) \sum_{\mathfrak{p}}\left(\left((N+1) / v_{\mathfrak{p}}\left(j_{\mathcal{E}}^{-1}\right)\right)-v_{\mathfrak{p}}\left(j_{\mathcal{E}}^{-1}\right)\right)$, where where $\sum_{\mathfrak{p}}$ denotes the sum over $\mathfrak{p} \in M_{K}$ such that $v_{\mathfrak{p}}\left(j_{\mathcal{E}}\right)<0$. Since $\sum_{\mathfrak{p}}^{\prime} v_{\mathfrak{p}}\left(j_{\mathcal{E}}^{-1}\right)=$ $d_{E / K}$ and $\left|\left\{\mathfrak{p} \in M_{K} ; v_{\mathfrak{p}}\left(j_{\mathcal{E}}\right)<0\right\}\right|=f_{E / K}, H \geqslant(1 /(48 N))\left((N+1) d_{E / K} \sigma_{E / K}^{-2}-d_{E / K}\right)$. By hypothesis $N+1>2 \sigma_{E / K}^{2}$, therefore $H>d_{E / K} \sigma_{E / K}^{-2} / 96$.

Corollary 4. For every $P \in E(K)$ of infinite order we have $\widehat{h}(P) \geqslant\left(d_{E / K}\right.$ $\left.\sigma_{E / K}^{-6}\right) / 1536$.

Proof: Suppose that $\widehat{h}(P)<d_{E / K} \sigma_{E / K}^{-6} / 1536$. For any integer $n$ such that $1 \leqslant$ $n \leqslant 4 \sigma_{E / K}^{2}, \widehat{h}(n P)=n^{2} \widehat{h}(P)=d_{E / K} \sigma_{E / K}^{-2} / 96$. But this shows that $\left|\mathcal{S}_{\sigma}\right| \geqslant 4 \sigma_{E / K}^{2}$, which contradicts Proposition 3.

Corollary 4 implies the following version of a conjecture of Lang (see [3, Theorem $0.2])$.

ThEOREM 5. For every $P \in E(K)$ of infinite order there exists a constant $c_{2}\left(j_{\mathcal{E}}, g\right)$ depending on $g$ and on the inseparable degree $p^{e}$ of $j_{\mathcal{E}}: X \rightarrow \mathbb{P}_{k}^{1}$ such that $\widehat{h}(P) \geqslant$ $c_{2}\left(j_{\mathcal{E}}, g\right) d_{E / K}$, where $c_{2}\left(j_{\mathcal{E}}, g\right)$ is equal to $\left((2.18) 10^{-10}\right) p^{-6 e}$, if $d_{E / K} \geqslant 24 p^{e}(g-1)$ and to $\left((3.4) 10^{-12}\right) p^{-6 e} g^{-6}$, if $d_{E / K}<24 p^{e}(g-1)$. 
Proof: Szpiro's theorem on the minimal discriminant of elliptic curves over function fields states that $d_{E / K} \leqslant 6 p^{e}\left(2 g-2+f_{E / K}\right)$ (see [9, Théorème 1]). Hence, $\sigma_{E / K}^{-1} \geqslant\left(6 p^{e}\right)^{-1}-(2 g-2) d_{E / K}^{-1}$. In the case where $d_{E / K} \geqslant 24 p^{e}(g-1)$, we obtain $\sigma_{E / K} \leqslant 12 p^{e}$. Otherwise, $\sigma_{E / K} \leqslant d_{E / K}<24 p^{e}(g-1)$. These two inequalities and Corollary 4 prove the theorem.

REMARK 6 . Theorem 5 slightly improves [3, Theorem 0.2$]$ in the sense that the lower bound for the canonical height of points of infinite order depends polynomially on $\sigma_{E / K}$, instead of exponentially. This had already been remarked and proved for elliptic curves over number fields by David (see [1, Corollaire 1.5]) using transcendence methods, which in contrast with Hindry-Silverman's method is global rather than local.

As a consequence of Proposition 3 we obtain an upper bound for the torsion subgroup $E(K)_{\text {tor }}$ of $E(K)$.

THEOREM 7. $\left|E(K)_{\text {tor }}\right| \leqslant 2 \sigma_{E / K}^{2}$.

REMARK 8. In [2, Theorem 13] Goldfeld and Szpiro proved that $\left|E(K)_{\text {tor }}\right| \leqslant\left(6 p^{e}((2 g-\right.$ 2) $\left.\left.f_{E / K}^{-1}+1\right)\right)^{2}$. It follows from Szpiro's discriminant theorem that the bound of Theorem 7 is twice the bound of [2, Theorem 13]; however the method is different.

\section{INTEGRAL POINTS}

DEfinition 9: Let $R_{S} \subset K$ be the ring of $S$-integers and $R_{S}^{*} \subset R_{S}$ the group of $S$-units. Let $L$ be a finite extension of $K$ and $\alpha \in L$. Define $h_{L}(\alpha)=[L: k(\alpha)]$, if $\alpha \notin k$, otherwise $h_{L}(\alpha)=0$. Denote by $S_{L}$ the set of places of $L$ lying over $S$. Let $g_{L}$ be the genus of $L, R_{S_{L}} \subset L$ the ring of $S_{L}$-integers and $R_{S_{L}}^{*} \subset R_{S_{L}}$ the subgroup of $S_{L}$-units.

DEFINITION 10: Let $y^{2}=f(x)$ be a Weierstrass equation for $E / K$. Suppose that $f(X) \in R_{S}[X]$ and denote by $\Delta$ its discriminant. This equation is called $S$-minimal if $h_{K}(\Delta)$ is minimal subject to $f(X) \in R_{S}[X]$.

Definition 11: Let $f(X)=\left(X-\varepsilon_{1}\right)\left(X-\varepsilon_{2}\right)\left(X-\varepsilon_{3}\right)$ be the factorisation of $f(X)$ in $\bar{K}[X]$. Given $P=\left(x_{P}, y_{P}\right) \in E\left(R_{S}\right)$ and $i \in\{1,2,3\}$, let $\xi_{i}^{2}=x_{P}-\varepsilon_{i}$ and $L=K\left(\varepsilon_{1}, \varepsilon_{2}, \varepsilon_{3}, \xi_{1}, \xi_{2}, \xi_{3}\right)$. For any permutation $\{i, l, m\}$ of the elements of $\{1,2,3\}$, let $\Xi=\left\{\left(\xi_{i}-\xi_{l}\right) /\left(\xi_{i}-\xi_{m}\right),\left(\xi_{i}-\xi_{l}\right) /\left(\xi_{i}+\xi_{m}\right),\left(\xi_{i}+\xi_{l}\right) /\left(\xi_{i}-\xi_{m}\right),\left(\xi_{i}+\xi_{l}\right) /\left(\xi_{i}+\xi_{m}\right)\right\}$.

The main result needed to obtain an explicit bound for the number of $S$-integral points of an $S$-minimal Weierstrass equation for $E$ is an upper bound for the height of the $y$-coordinates of integral points (see [3, Proposition 8.2]). Before doing this it is necessary to obtain an upper bound for the height of $S$-units.

Proposition 12. Let $y^{2}=f(x)$ be a Weierstrass equation for $E / K$. Suppose that $f(X) \in R_{S}[X], \Delta \in R_{S}^{*}$ and $p>2$. For any $\eta \in \Xi$ we have $h_{L}(\eta) \leqslant 2 p^{e}\left(2 g_{L}-2+\right.$ $\left.\left|S_{L}\right|\right)$. 
PROOF: Let $t=\left(\varepsilon_{3}-\varepsilon_{1}\right) /\left(\varepsilon_{2}-\varepsilon_{1}\right)$ and denote by $y^{2}=x(x-1)(x-t)$ a Legendre form of $E / K$. Note that since the inseparable degree of $j_{\mathcal{E}}$ is $p^{e}, j_{\mathcal{E}} \in K^{p^{e}}-K^{p^{e+1}}$. But $j_{\mathcal{E}}=2^{8}\left(t^{2}-t+1\right)^{3} /\left(t^{2}(t-1)^{2}\right)$, thus $t \notin L^{p^{2+1}}$. Furthermore, any permutation of 1,2 and 3 replaces $t$ by an element of $\{t, 1-t, 1 / t, 1 /(t-1), t /(t-1),(t-1) / t\}$. Therefore, for any distinct $i, l, m \in\{1,2,3\}, \kappa=\left(\varepsilon_{l}-\varepsilon_{i}\right) /\left(\varepsilon_{m}-\epsilon_{i}\right)=\left(\left(\xi_{i}-\xi_{l}\right) /\left(\xi_{i}-\xi_{m}\right)\right)\left(\left(\xi_{i}+\right.\right.$ $\left.\left.\xi_{l}\right) /\left(\xi_{i}+\xi_{m}\right)\right) \notin L^{p^{e+1}}$. Suppose that for any $\eta \in \Xi$ we have $\eta \notin L^{p^{e+1}}$. Let $0 \leqslant r, s \leqslant e$ be the smallest integers such that $\kappa \in L^{p^{r}}-L^{p^{r+1}}$ and $\eta \in L^{p^{s}}-L^{p^{s+1}}$, respectively. Denote $\kappa_{r}=\kappa^{p^{r}}$ and $\eta_{s}=\eta^{p^{a}}$. Observe that $\kappa_{r}, 1-\kappa_{r}, \eta_{s}, 1-\eta_{s} \in R_{S_{L}}^{*} \cap\left(L-L^{p}\right)$. It follows from [6, Chapter VI, Lemma 10] that $h_{L}\left(\kappa_{r}\right), h_{L}\left(\eta_{s}\right) \leqslant 2 g_{L}-2+\left|S_{L}\right|$. Hence, $h_{L}(\kappa), h_{L}(\eta) \leqslant p^{e}\left(2 g_{L}-2+\left|S_{L}\right|\right)$. If some $\eta \in \Xi$ lies in $L^{p^{e+1}}$, then $\tau=\kappa \eta^{-1} \notin L^{p^{c+1}}$. By using the same argument as above we conclude that $h_{L}(\tau) \leqslant p^{e}\left(2 g_{L}-2+\left|S_{L}\right|\right)$. Therefore, $h_{L}(\eta)=h_{L}(\kappa)+h_{L}(\tau) \leqslant 2 p^{e}\left(2 g_{L}-2+\left|S_{L}\right|\right)$, which proves the proposition. $\quad$

Proposition 13. With the same hypothesis and notation of Proposition 12, suppose furthermore that $p>3$. For any $P=\left(x_{P}, y_{P}\right) \in E\left(R_{S}\right)$ we have $h_{K}\left(y_{P}^{4} / \Delta\right) \leqslant$ $48 p^{e}(2 g-2+|S|)$.

Proof: The proof follows the same lines as [3, Proposition 8.2] replacing [3, (42)] by the inequality of Proposition 12. However, we need to remark that the RiemannHurwitz formula can be applied for $L / K$, because $p>3$ implies that $L / K$ is separable and has no wild ramification.

In order to obtain an explicit upper bound for $\left|E\left(R_{S}\right)\right|$, recall from [7, Lemma 1.2 (a)] that $\left|E\left(R_{S}\right)\right| \leqslant\left|E(K)_{\mathrm{tor}}\right|(1+2 \sqrt{\beta / \alpha})^{r_{E}}$, where $\alpha=\min \{\widehat{h}(P) ; P \in(E(K)-$ $\left.\left.E(K)_{\text {tor }}\right) \cap E\left(R_{S}\right)\right\}, \beta=\max \left\{\widehat{h}(P) ; P \in E\left(R_{S}\right)\right\}$ and $r_{E}=\operatorname{rank}(E(K))$. The lower bound $\alpha$ is obtained from Theorem 5 .

REMARK 14. Since $p>3$, we write the Weierstrass equation of $E / K$ as $y^{2}=x^{3}+$ $A x+B$. Suppose it is $S$-minimal. In this case, $\beta \leqslant p^{e}\left(12 g+4|S|+5 d_{E / K}\right)$. The proof of this inequality is the same as in [3, Corollary 8.5] replacing [3, Proposition 8.2] by Proposition 13.

THEOREM 15. Suppose that $p>3$ and $y^{2}=x^{3}+A x+B$ is an S-minimal equation for $E / K$. If $d_{E / K} \geqslant 24 p^{e}(g-1)$, then $\left|E\left(R_{S}\right)\right| \leqslant 288 p^{2 e}\left(\left((8.57) 10^{5}\right) p^{4 e} \sqrt{|S|}\right)^{r_{E}}$; otherwise $\left|E\left(R_{S}\right)\right| \leqslant(1152) p^{2 e} g^{2}\left(\left((2.51) 10^{7}\right) p^{4 e} g^{4} \sqrt{|S|}\right)^{r_{E}}$.

ProOF: Suppose that $d_{E / K} \geqslant 24 p^{e}(g-1)$. Thus $g \leqslant d_{E / K} p^{-e} / 24+1$ and $\sigma_{E / K} \leqslant$ $12 p^{e}$. Since $|S| \geqslant 1, \beta / \alpha \leqslant\left((4.59) 10^{9}\right) p^{7 e}\left(5+(12 g+4|S|) d_{E / K}^{-1}\right) \leqslant\left((4.59) 10^{11}\right) p^{7 e}|S|$. Theorem 7 implies $\left|E(K)_{\text {tor }}\right| \leqslant 2 \sigma_{E / K}^{2} \leqslant 288 p^{2 e}$. This proves the first part of the theorem. Suppose now that $d_{E / K}<24 p^{e}(g-1)$. In this case, since $|S| \geqslant 1$ and $g \geqslant 2$, $\beta / \alpha \leqslant\left((2.94) 10^{11}\right) p^{7 e} g^{6}\left(5+(12 g+4|S|) d_{E / K}^{-1}\right) \leqslant\left((3.94) 10^{13}\right) p^{7 e} g^{7}|S|$. It follows from 
Theorem 7 that $\left|E(K)_{\text {tor }}\right| \leqslant 2 \sigma_{E / K}^{2}<2\left(24 p^{e}(g-1)\right)^{2}$. Hence, the second part of the theorem is proved.

REMARK 16. Theorem 15 is an analogue for $\operatorname{char}(k)=p>3$ of $[3$, Theorem 8.1].

\section{REFERENCES}

[1] S. David, 'Points de petite hauteur sur les courbes elliptiques', J. Number Theory 64 (1995), 104-129.

[2] D. Goldfeld and L. Szpiro, 'Bounds for the Tate-Shafarevich group', Compositio Math. 86 (1995), 71-87.

[3] M. Hindry and J. Silverman, 'The canonical height and integral points on elliptic curves', Invent. Math. 93 (1988), 419-450.

[4] M. Hindry and J. Silverman, 'On Lehmer's conjecture for elliptic curves', in Séminaire de Théorie des Nombres Paris 1988-89 (1989), Progr. Math. 91 (Birkhaüser, Boston, MA, 1990), pp. 103-116.

[5] S. Lang, Fundamentals of diophantine geometry (Springer-Verlag, Berlin, Heidelberg, New York, 1983).

[6] R.C. Mason, Diophantine equations over function fields, Lecture Notes of the London Math. Soc. 96 (Cambridge University Press, Cambridge, London, 1984).

[7] J. Silverman, 'A quantitative version of Siegel's theorem: integral points on elliptic curves and Catalan curves', J. Reine Angew. Math. 378 (1987), 60-100.

[8] J. Silverman, Advanced topics in the arithmetic of elliptic curves (Springer-Verlag, Berlin, Heidelberg, New York, 1994).

[9] L. Szpiro, 'Discriminant et conducteur d'une courbe elliptique', Astérisque 86 (1990), 7-18.

[10] J.F. Voloch, 'Explicit p-descent for elliptic curves in characteristic p', Compositio Math. 74 (1990), 247-258.

Rua Guaiaquil 83

Cachambi

20785-050 Rio de Janeiro, RJ

Brasil

e-mail: amilcar@impa.br 